\title{
A computational framework for the analysis of rain-induced erosion in wind turbine blades, part I: stochastic rain texture model and drop impact simulations
}

\author{
B. Amirzadeh ${ }^{1}$, A. Louhghalam ${ }^{1}$, M. Raessi ${ }^{1}$, M. Tootkaboni ${ }^{1, *}$ \\ University of Massachusetts Dartmouth, 285 Old Westport Rd. North Dartmouth MA, USA
}

\begin{abstract}
In the past decade, the power output of wind turbines has increased significantly. This increase has been primarily achieved through manufacturing larger blades resulting in high blade tip velocities and increased susceptibility to rain erosion. This paper is the first part in a two-part paper that presents a framework for the analysis of rain erosion in wind turbine blades. Two ingredients of the framework are presented. A stochastic rain texture model is developed to generate three-dimensional fields of raindrops consistent with the rainfall history at the turbine location by integrating the micro-structural properties of rain, i.e. raindrops size and spatial distribution with its integral properties such as the relationship between the average volume fraction of raindrops and rain intensity. An in-house GPU-accelerated computational fluid dynamics model of free-surface flows and a multi-resolution strategy are used to calculate the drop impact pressure as a function of time and space. An interpolation scheme is finally proposed to find the time evolution of impact pressure profile for any given drop diameter using the high fidelity simulation results, significantly reducing the computational cost. Other ingredients of the framework pertaining to drop impact-induced stresses and the blade coating fatigue life are presented in part II.
\end{abstract}

Keywords: wind turbine blade, rain-induced erosion, stochastic rain texture, drop impact simulation, multi-resolution analysis

\section{Introduction}

Along with the growing interest in renewable energies as a substitute for fossil fuels, there has been an increasing effort in the wind energy industry to increase the power output of wind turbines. In fact, in the past decade, the world has witnessed a ten-fold increase in the amount of power generated from wind [1]. It is also predicted that 8 to $12 \%$ of the world's total energy will be provided by wind power by the year 2020 [2]. This has fueled the production of wind turbines with higher power output in recent years. As is evident from the specifications of these wind turbines [3, 4], increasing the blade size is the primary means of increasing the turbines' power output (see Figure 1). Compared to

\footnotetext{
${ }^{*}$ Corresponding author

Email addresses: bamirzadeh@umassd.edu (B. Amirzadeh), alouhghalam@umassd.edu (A. Louhghalam), mraessi@umassd.edu (M. Raessi), mtootkaboni@umassd.edu (M. Tootkaboni)

${ }^{1}$ University of Massachusetts Dartmouth

Preprint submitted to Journal of Wind Engineering $\mathcal{E}$ Industrial Aerodynamics

November 18, 2016
} 
the wind turbines of 1980s, modern versions are much bigger and have rotor blades that are up to eight times larger in diameter. As a result, the blade tip velocity of the largest wind turbines can be as high as $120 \mathrm{~m} / \mathrm{s}$ (see Figure 2), while other sources report even higher velocities around 180 to $200 \mathrm{~m} / \mathrm{s}$ [5, 6]. With such high blade tip velocities comes higher susceptibility to erosion, particularly in harsh climates where heavy rain and hail are common [7, 8].

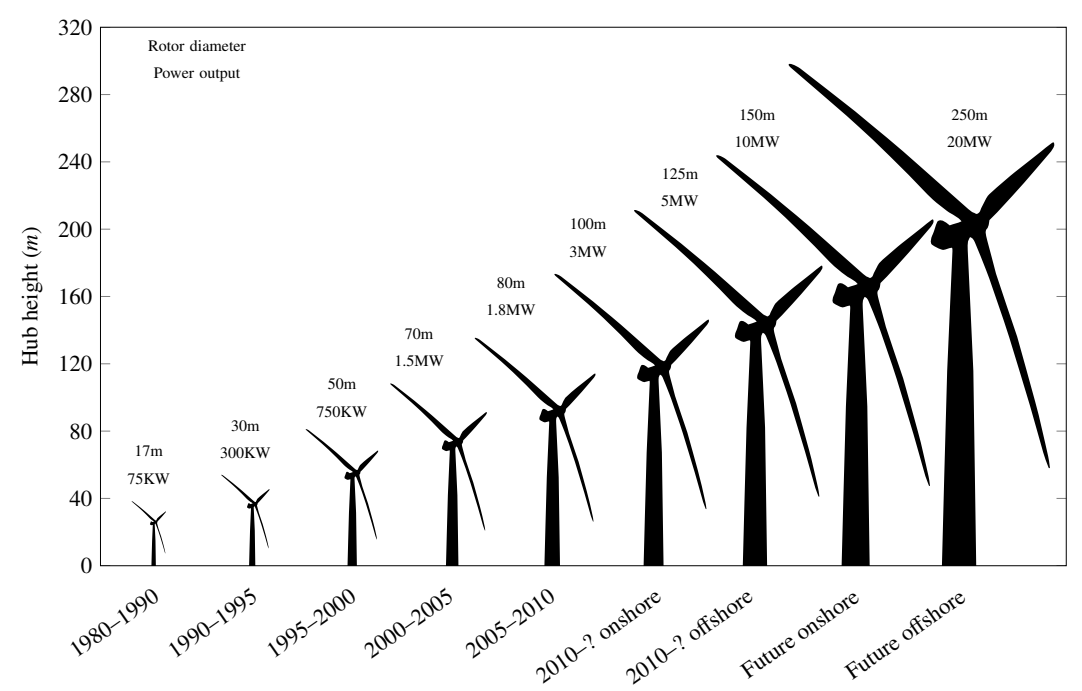

Figure 1: Current trend in wind turbine size. Adapted from [9].

Rain erosion of the blades begins with a gradual increase in blade's surface roughness until small pits form near the leading edge. Over time, the density of these pits increases until gouges are formed [10]. However, performance degradation is initiated in the early stages of erosion, where the surface roughness starts to increase. A rougher surface increases the aerodynamic drag coefficient of the blades, which results in an undesirably lower performance and energy loss. For example, in a 2.5 MW wind turbine, simulated erosion experiments have shown that leading edge erosion can severely affect the airfoil performance to such an extent that, depending on the severity of erosion, up to $25 \%$ annual energy loss can be expected [10]. Other studies have confirmed the impact of surface roughness on aerodynamic force coefficients and stall characteristics $[11,12,13,14]$ and consequently on aerodynamic performance $[15,16]$ as well as dynamic instabilities [17]. Evidence of leading edge erosion can be found on the blades' surface in as early as two to three years after installation [5, 18]. If the blades are not repaired on time, the underlying composite laminates will also be affected. At this point, the blades have to be taken down for extensive maintenance or even replacement, both of which are costly operations [18, 19]. Regardless of the strategy adopted to prevent energy loss due to loss of aerodynamic performance, deterioration mechanisms such as blade surface erosion are associated with significant uncertainty and need to be taken into account in the planning of the operation and maintenance as well as reliability analysis procedures [20, 21]. 


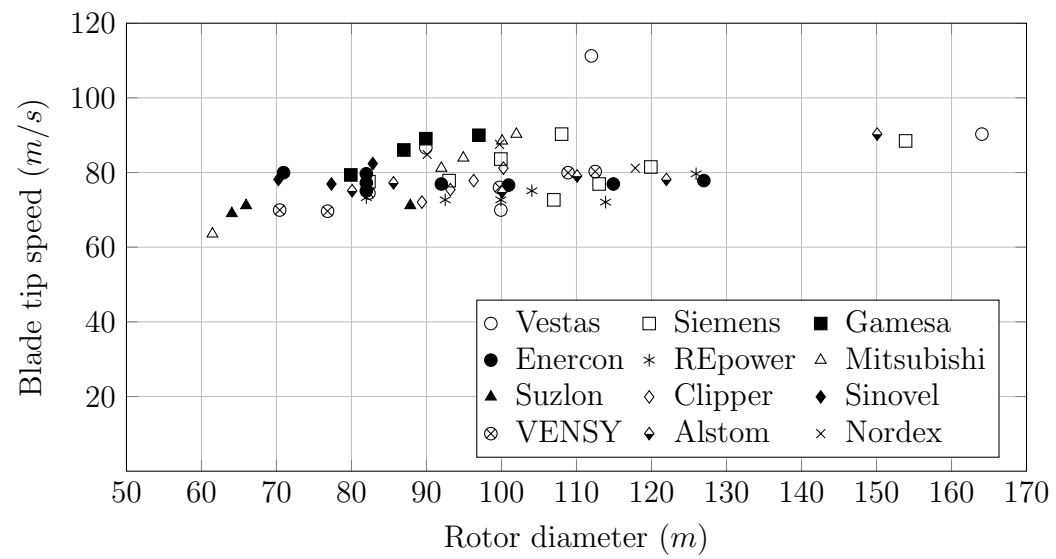

Figure 2: Maximum blade tip velocity vs. rotor diameter from several wind turbine manufacturers [22].

Fiber reinforced composites have long been the materials of choice in the wind turbine industry due to their high stiffness and low weight. They perform excellently over large length scales of the blade, however, they do not perform as well under transverse impact loads and are prone to degradation by environmental factors. Therefore, manufacturers have put considerable emphasis on developing protective coatings for wind turbine blades. These coatings are usually referred to as "Gelcoat" in the industry and they serve to protect the underlying composite laminates from weathering and foreign object impact [23]. Leading edge tapes have also found their spot particularly in repair operations. They are usually made of highly elastic and durable polyurethane material to act as an energy absorber in impacts [22]. However, experiments have shown that leading edge erosion protection tapes can also be detrimental to the turbine aerodynamic performance [24].

Throughout the years, in order to assess the rain erosion resistance of different materials, many rain erosion testing techniques have been developed. These include on-site testing, whirling arms, single and multiple impact waterjets, single droplet impact and rocket sled tests. However, these methods have limitations in various aspects such as cost, reproducibility and drop size variations and may lead to unrealistic forces and boundary conditions. In addition, due to the complexity of multiple particle erosion scenarios, analytical predictive models have been mainly limited to single impact cases [25]. In fact, although blade surface quality in wind turbines can seriously affect their performance, there has not been much effort on creating a holistic view of rain-induced erosion in wind turbine blades. According to Dalili [7], this is most likely due to the complexity and the multifaceted nature of blade surface erosion phenomena. Overall, there is no consensus as to whether a realistic study of multiple impact erosion is feasible or not. Some researchers have completely dismissed the option of analytical or numerical solutions and have adopted a fully probabilistic approach [26], while others have proposed expensive explicit dynamics simulations as a viable choice for the simulation of repeated impact scenarios [27] and protective coating evaluations [28]. Above all, it is apparent that raindrop impingement erosion should be looked at as a system's property rather than a material's property, in which the operational conditions of the wind turbine are taken into account as well [29]. From this viewpoint, there 
has not been many investigations until recently where only general analytical models of erosion phenomena have been discussed [30]. Specifically, a detailed model of rain is absent in structural mechanics and erosion studies and most researchers have been content with overly simplified rain load models [31].

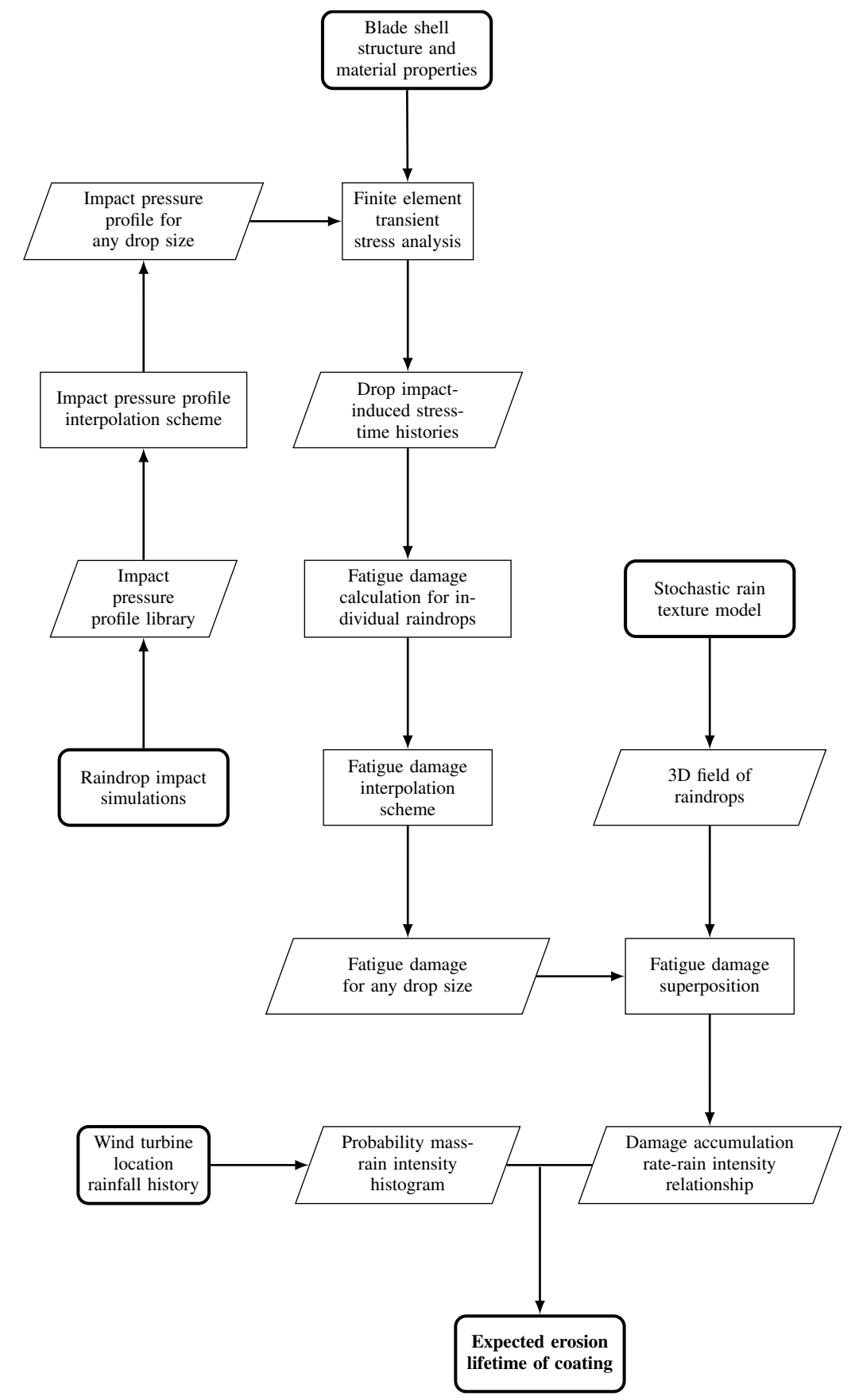

Figure 3: The ingredients of the proposed computational framework.

This paper is the first part in a two-part paper that presents a computational framework for rain erosion prediction 
with application to wind turbine blades. Rather than a detailed analysis of the individual aspects of rain erosion, the goal is to integrate well established theories and models to build a framework that can be used to estimate the expected erosion lifetime of the blade, given the rainfall history at a particular location, the blade shell characteristics (layup structure, mechanical properties) and the operational conditions of the wind turbine. The lifetime is defined as the time it takes for the blade coating to develop aerodynamically undesirable roughness known as the incubation period. The proposed framework consists of several components based in probability theory and stochastic processes, computational fluid dynamics, finite element modeling and fatigue analysis (see Figure 3, see also [32]). We begin by looking at the rainfall history in the wind turbine location. Specifically, the probability mass of all possible rain intensities is calculated by averaging over a number of annual rain intensity-duration graphs. Subsequently, a rain texture model is developed that allows for the transformation of the rain intensity values into three dimensional fields of raindrops. The next step, which lies at the core of the erosion life estimation, involves the calculation of the fatigue damage for each raindrop by performing a stress analysis of its collision with the coating surface. The stresses are calculated through finite element modeling of drop impact, in which the droplet impact pressure is applied on the surface as a spatially varying time dependent external load. These impact pressure profiles are calculated by an interpolation scheme which uses a library of impact pressure profiles obtained by modeling the surface impact of rain drops of various diameters via high-fidelity GPU-accelerated CFD simulations. In part I of this paper, we discuss the rainfall data analysis, the development of the stochastic rain texture model, multi-resolution droplet impact CFD simulations and the development of the impact pressure profile interpolation scheme, while raindrop impact-induced transient stress analysis and the fatigue damage calculations are discussed in part II [32]. It is finally noted that the individual components of the proposed framework can be potentially extended to other rain erosion scenarios as well.

\section{A stochastic model of rain texture}

From a meteorological perspective, a rain event is characterized by its intensity and duration. The intensity of rain is measured by rain gauges and is expressed as the height of the water accumulated in a certain time period, usually in $\mathrm{mm} / \mathrm{hr}$. However, for the processes where the effects of individual raindrops are of interest, an intensity value per se is not very useful. In these cases, a more detailed model of the rain has to be considered; one that involves both the sizes of the raindrops and their distribution in space (see e.g. [33]). More importantly, this model should be able to relate these characteristics to the rain intensity. In what follows, we discuss the steps involved in creating such a model, which ultimately defines what is usually referred to as rain micro-structure or rain texture.

\subsection{Rain intensity data}

One of the most common ways to present rainfall data is in the form of the rain intensity-duration. Several factors may affect the choice of timespan when analyzing rainfall data. First and foremost, the timespan of the rain data has to be large enough to account for the statistical variability when calculating the average annual rain scenario. The 
other factor that needs to be taken into account is the expected lifetime of a wind turbine which is around 20 to 25 years. The average annual rain scenario to be used in a wind turbine blade erosion study, better be calculated from a timespan which is comparable to this lifetime. Finally, the availability of data for the location of interest is also an important factor. In the current study, the rainfall data for the city of New Bedford between the years 1992 and 2000 is used. The data is provided by NOAA's National Climatic Data Center (NCDC) [34]. The probability mass function of the combined data for the 9-year timespan is shown in Figure 4. Another important piece of information that we extract from this data is the number of raining hours per year. This number (or its average) will be used in the final stage for the purpose of fatigue life estimation.

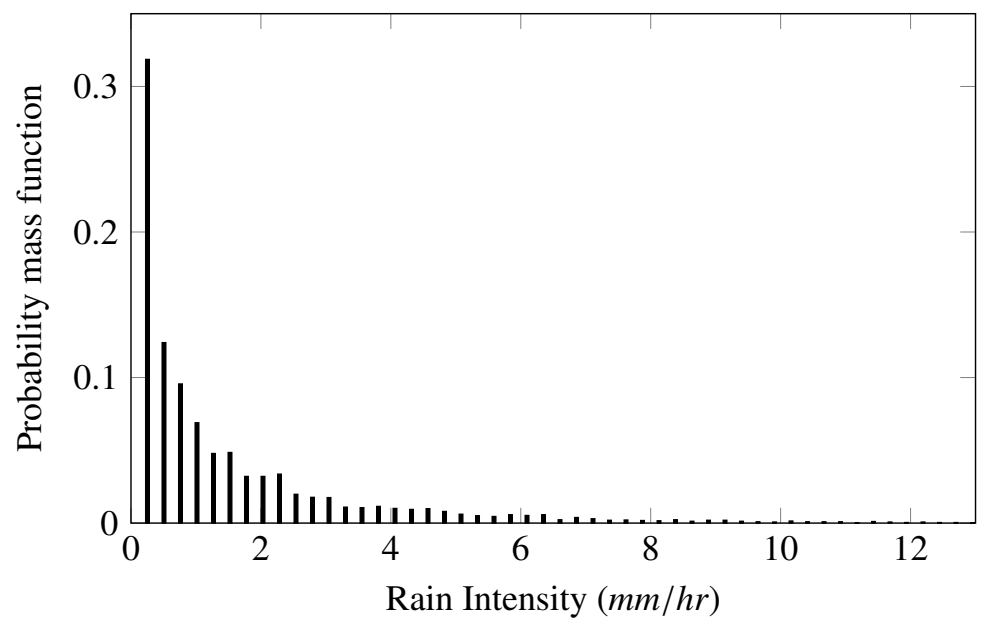

Figure 4: The probability mass function (PMF) of rain intensities in New Bedford, MA from 1992 to 2000.

\subsection{Raindrop size distribution}

As mentioned earlier, the role of a rain texture model is to relate the rain intensity values with the size and position of raindrops. Among many studies on the raindrop size distribution [35, 36, 37, 38, 39], one of the most comprehensive is the work of Best [35]. Best proposed a relationship for the raindrop size distribution that closely matches the experimental data from other sources such as Marshall and Palmer [36]. Best's drop size distribution has the following form:

$F=1-\exp \left[-\left(\frac{d}{1.3 I^{0.232}}\right)^{2.25}\right]$

where $d$ is the drop diameter in $m m, I$ is the rain intensity in $m m / h r$ and $F$ is the fraction of liquid water in air comprised of drops with a diameter smaller than $d$. In statistical terms, $F$ can be interpreted as the cumulative distribution function of raindrop size. The effect of rain intensity on raindrop size can be understood clearly by visualizing the associated probability density function. This function is plotted at various rain intensities in Figure 5. 


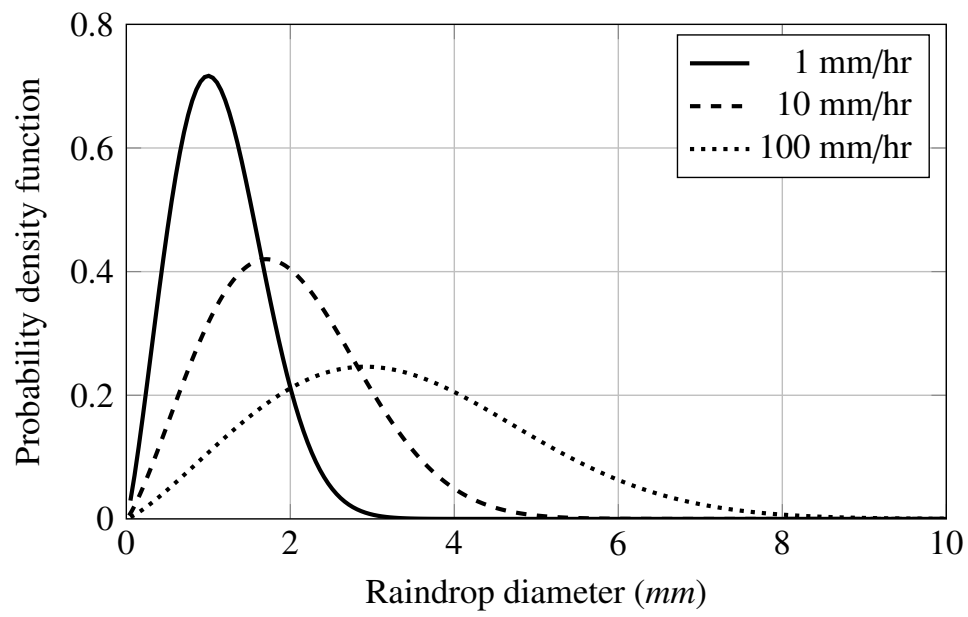

Figure 5: Probability density function (PDF) of raindrop size at various rain intensities derived from Equation (1).

By sampling from the distribution given in Equation (1), it is possible to generate samples of raindrop sizes in a unit volume of air. A constraint that helps determine the number of drops is the total volume of water per unit volume of air. Best [35] suggested the following relationship between the volume of water in air and the rain intensity:

$W=67 I^{0.846}$

where $I$ has the same definition as in Equation (1) and $W$ is the amount of liquid water per unit volume of air expressed in $\mathrm{mm}^{3}$ of water per $\mathrm{m}^{3}$ of air. With the volume of water-intensity relationship given in Equation (2) all that is missing from the rain texture model is the spatial distribution of the raindrops. In what follows, we try to provide this missing piece by relying on the fact that the spatial distribution of raindrops often resembles a state of perfect randomness.

\subsection{A compound stochastic point process for spatial distribution of raindrops}

Meteorological studies mostly focus on integral properties of rain at length scales on the order of several kilometers. This allows for a continuum view of rain. However, at length scales comparable to the distance between the raindrops, a discrete view is more appropriate and the rain can be considered as a stochastic point process. From this perspective, a widely used assumption about the micro-structure of rain in the literature is considered. This assumption considers rain to be perfectly random, spatially and temporally. In fact, many sources view rain as a typical example of a homogeneous spatial Poisson process, which exhibits complete spatial randomness [40] (see also [41] for a discussion). What this means is that the number of drops in volume $V, N(V)$, follows a Poisson distribution:

$P(N(V)=k)=\frac{(\lambda V)^{k} e^{-\lambda V}}{k !}$

with $\lambda$ the expected number of raindrops in volume $V$ and $P(N(V)=k)$ the probability of finding $k$ raindrops in volume $V$. It also means that the drops are uniformly distributed in space. It should be noted that real rain is not 
necessarily always a perfectly random process. In other words, the presence of a raindrop at a specific point can affect the probability of finding other raindrops nearby. This, at the least, violates the independence assumption inherent in the definition of spatial Poisson process. Consequently, raindrop clustering can occur, which leads to patchiness in the rain texture. While models for spatial correlations between raindrops have been proposed elsewhere [42, 41], the length scale of such events (on the order of tens of milliliters) is significantly larger than the length scale over which one may observe interactions among individual drops' impact-induced stresses within the blade coating (on the order of milliliters); see part II [32]. Therefore in this study, we assume that the effects of clustering events do not play a key role and we adopt a model of perfect randomness for the rain texture.

In order to close the rain texture model, let us recapitulate three key points about rain micro-structure discussed so far: (i) the distribution of the size of raindrops follows Equation (1) (ii) the volume of liquid water per unit volume of air follows Equation (2) (iii) the number of drops in unit volumes of air follows a Poisson distribution (see Equation (3) for $V=1$ ), and the raindrops are uniformly distributed in space. Having Equations (1) to (3) and given a rain intensity $I$, the only missing piece is the rate parameter $\lambda$ in Equation (3). In order to find $\lambda$ for a given rain intensity, a simple trial and error search technique is used, the details of which are explained here.

Consider a rain intensity $I$, a spatial domain of $Q \in \mathbb{N}$ unit volumes and a range of $\lambda$ values defined as

$\Lambda=\left\{\lambda \mid \lambda \in\left[\lambda_{\min }, \lambda_{\max }\right] ; \lambda_{\min }, \lambda_{\max } \in \mathbb{N}\right\}$

to scan for the desired value. From Equation (3), the number of drops per unit volume of air follows a Poisson distribution. Starting with $\lambda_{\min }, Q$ samples are generated from this distribution. The resulting set contains the number of raindrops per unit volume of space for all the $Q$ unit volumes:

$G=\left\{g_{i} \mid i \in[1, Q]\right\}$

where the subscript $i$ indicates the $i$ th unit volume. The next step is to assign sizes to the raindrops within each unit volume. In order to do so, the size distribution given by Equation (1) is sampled. The inverse transform sampling technique is used for this purpose, which is readily applicable when the cumulative distribution function is known [43]. The resulting set $R$ contains drop sizes for the raindrops filling the $Q$ unit volumes:

$R=\left\{r_{i, j} \mid i \in[1, Q] ; j \in\left[1, g_{i}\right] ; r_{i, j} \in \mathbb{R}^{+}\right\}$

where the subscript $j$ indicates the $j$ th raindrop in the $i$ th unit volume. 


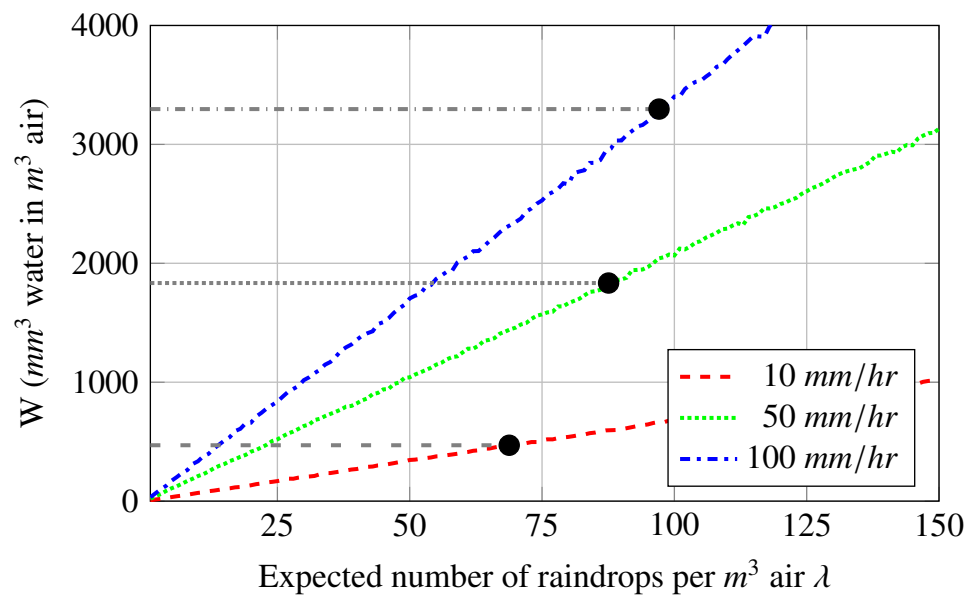

Figure 6: Variations of the expected volume of water per unit volume of air as a function of the expected number of raindrops in unit volume of air. The dashed horizontal lines indicate the the value for $W$ given by Equation (2). The black circles correspond to the value of $\lambda$ which satisfies this $W$.

Knowing the size of the generated raindrops, the next step is to calculate the volume of water $W$, in each of the $Q$ unit volumes of air. The expected value of $W$ is then calculated by averaging over all the $W$ values associated with the $Q$ unit volumes. This value is then checked against that given by Equation (2). The aforementioned procedure is repeated by iterating over all values of $\lambda$ in the set $\Lambda$ until a matching $\lambda$ is found. This is shown in Figure 6 for three different rain intensities. Note that the choice of Q affects the accuracy of the calculated $\lambda$. This effect is shown in Figure 7 for three different rain intensities where it is seen that $\lambda$ converges to a constant value as the size of the sampling volume is increased.

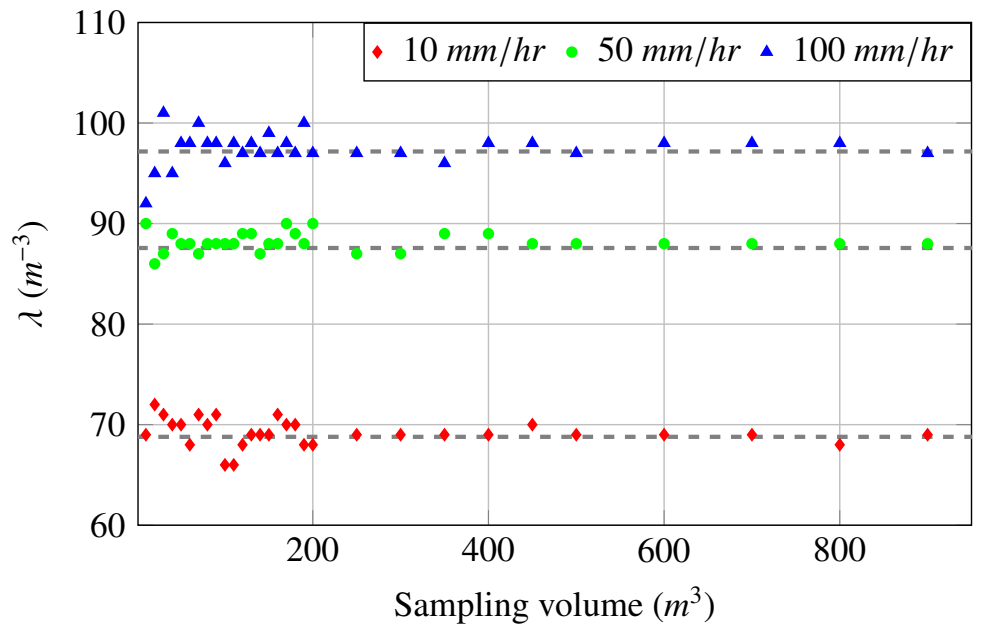

Figure 7: The effect of the sampling volume on the calculated $\lambda$ value. Notice that $\lambda$ converges as the sampling volume is increased. The dashed gray line for each rain intensity indicates the value from Equation (7).

Following the procedure described above, the expected number of raindrops per unit volume $\lambda$, can be calculated 
as a function of rain intensity. This relationship is shown in Figure 8 where it is seen that, similar to Equation (2), $\lambda$ has a power law relationship with $I$ as follows:

$\lambda=48.88 I^{0.15}$

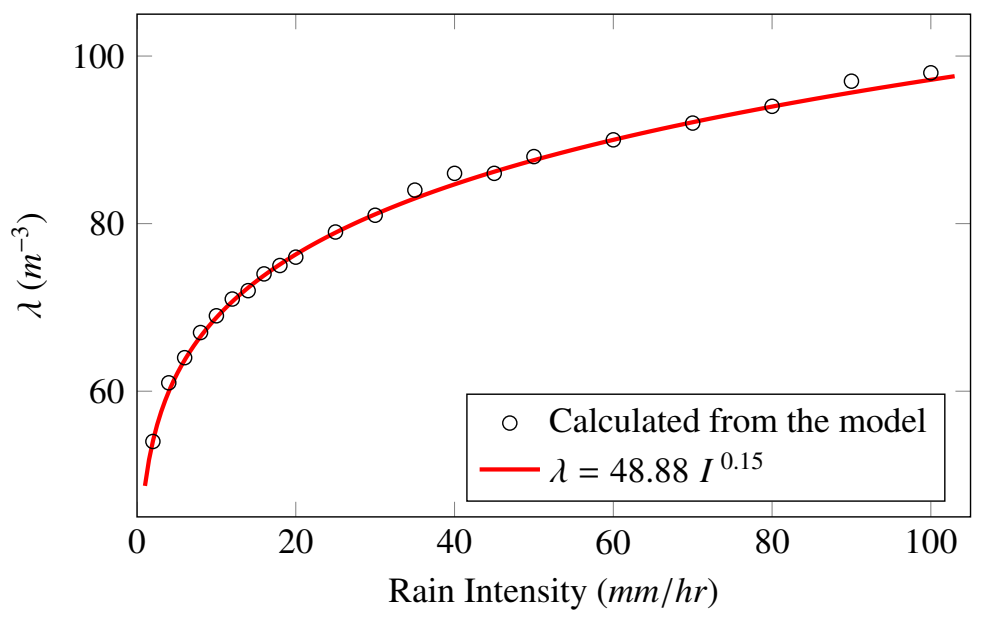

Figure 8: Expected number of raindrops per $m^{3}$ vs. rain intensity.

\subsection{Creating a rain scenario}

It would be helpful at this point to briefly explain how the rain texture model is used in connection with erosion prediction. As will be explained in part II of this paper [32], the erosion study is performed only on a small area that represents a portion of the blade shell. The impacts are all perpendicular to the plate and at constant velocity (the blade tip velocity) as we neglect other effects such as the raindrops' terminal velocity, impact angle and wind speed. In the light of this argument, it can be assumed that the raindrops are motionless and the plate is moving through space on a straight path and colliding with them. Therefore, when it comes to reproducing a rain event, it is convenient to think that the raindrops are distributed in a tall column with a unit base area above the plate. The height of this column is determined by the duration of the rain that we want to recreate and the blade tip velocity.

In order to explain the creation of a three dimensional field of raindrops an example scenario is provided herein. Consider a rain event with an intensity of $5 \mathrm{~mm} / \mathrm{hr}$ and a duration of 20 seconds. Also, assume that the impact velocities are $100 \mathrm{~m} / \mathrm{s}$. Since all raindrops have the same velocity, a 20 second rain for instance, requires a $2 \mathrm{~km}$ high column. First, the intensity value is plugged into Equation (7). The resulting $\lambda$ is then used in the Poisson distribution in Equation (3), which is subsequently sampled $Q$ times with $Q$ the number of unit volumes in the rain column. Recall that the raindrops are assumed to be uniformly distributed in space. Therefore, the next step is to assign the $(x, y, z)$ coordinates to the drops by uniformly distributing them in the space. The last step is to assign raindrop sizes which is done by generating samples from Equation (1). Figure 9 depicts the visualization of rain texture for three different rain intensities. According to Best [35], Equation (1) is accurate if the 5\% of water comprised of the largest drops 
and $10 \%$ of water comprised of the smallest drops is not considered. This correction is applied to the model, through the following steps. First, the list of raindrops in the aforementioned column is sorted by drop size. Assuming that raindrops are perfectly spherical, this list is then converted to a drop volume list, the cumulative sum of which is also calculated. Those indices from the drop volume list that correspond to the $10 \%$ and $95 \%$ of the total volume are then identified. Finally, the drop sizes matching these indices are found in the sorted drop size list. These two raindrop sizes are the lower and upper limits which are then used to filter the drops list.

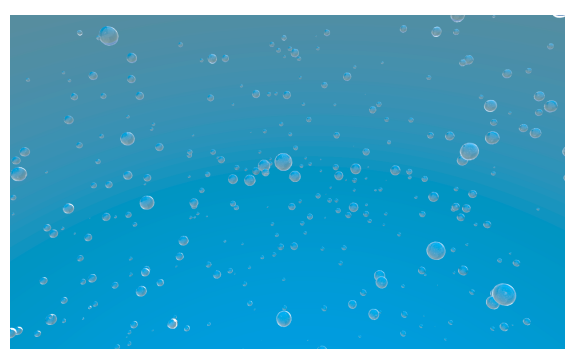

$1 \mathrm{~mm} / \mathrm{hr}$

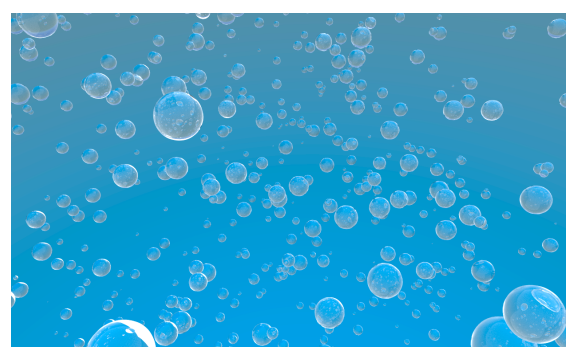

$10 \mathrm{~mm} / \mathrm{hr}$

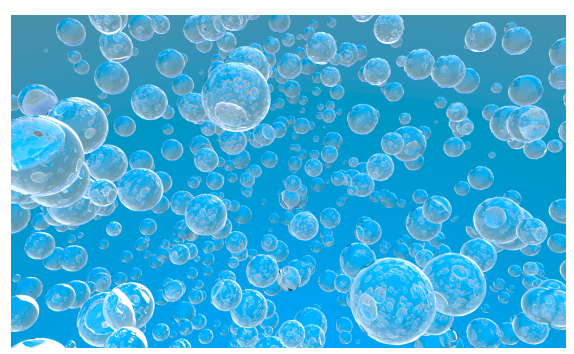

$100 \mathrm{~mm} / \mathrm{hr}$

Figure 9: Comparative rain texture visualization for 1, 10 and $100 \mathrm{~mm} / \mathrm{hr}$ rain intensities

\section{Liquid drop impingement erosion and CFD simulation}

The normal impact of a spherical liquid drop on a solid surface can be divided into several stages in time as shown in Figure 10, which illustrates a typical time history of the pressure induced due to drop impact. Immediately after the impact $\left(t<t_{1}\right)$, there is a rise in pressure on the central axis of the drop during which there is no considerable fluid flow. During this period, the maximum impact pressure remains on the central axis. The second stage $\left(t_{2}\right)$ is characterized by a pressure peak at the circumference of the contact circle between the drop and the solid. The maximum impact 
pressure is observed in this stage. Also, it is during this period that high speed lateral jets flow radially outwards from the base of the drop. Note that these features appear only several nanoseconds after impact. In the third stage $\left(t>t_{3}\right)$, the pressure on the solid surface gradually drops down to the stagnation pressure and eventually goes down to zero as the drop spreads on the surface.

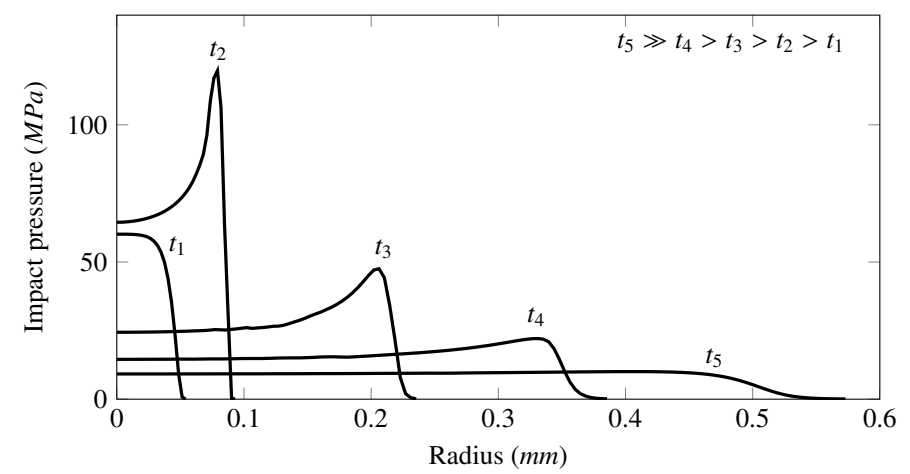

Figure 10: Radial distribution of the impact pressure at different stages of impact. The impact velocity is $100 \mathrm{~m} / \mathrm{s}$ and normal to the surface and the drop diameter is $1 \mathrm{~mm}$.

The progression of the liquid impact erosion phenomena can be expressed by measuring mass loss over time as shown schematically in Figure 11. During a time period after the start of the erosion process, there is no observable mass loss in the target. This period is known as the incubation period. In the second stage, the increasing period, mass loss begins and accelerates. This stage is followed by the placid period during which mass loss rate is reduced.

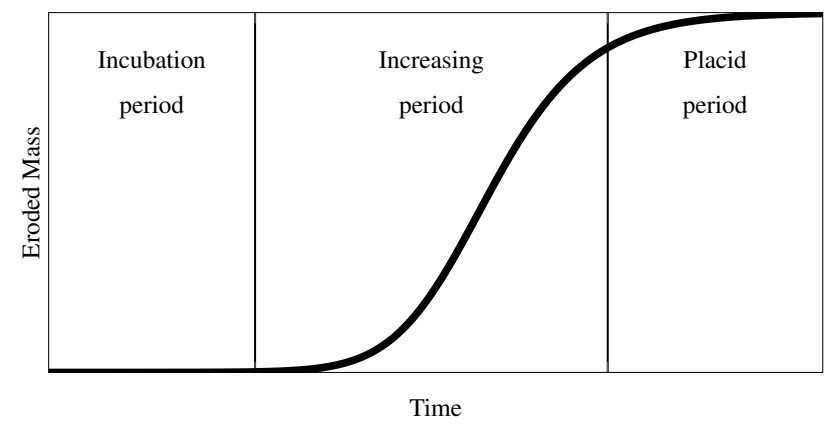

Figure 11: Mass loss vs. time in liquid impact erosion. Adapted from [44].

During the impact between a spherical drop and a solid surface, depending on the surface quality, different erosion mechanisms can be active. Depending on the characteristics of the target, particularly its surface quality, these mechanisms can be divided into four main categories: (a) direct deformation (b) stress wave propagation (c) lateral outflow jetting (d) hydraulic penetration [45]. In the incubation period, when the surface is still smooth, the damage mechanisms are restricted to direct deformation and stress wave propagation [46]. The damage in this stage is mainly attributed to the fatigue of the solid target material. However, as the erosion process continues and the surface roughness is increased, the lateral jetting and hydraulic penetration mechanisms are activated. In fact, in the increas- 
ing period, these mechanisms are the main contributors to erosion, mostly through shear-induced damage and crack opening [47]. When encountered by surface asperities, the high speed lateral jets can cause large shear stresses on the surface. Hydraulic penetration occurs when there are open cracks on the solid surface. High speed liquid can flow into these cracks and create high pressures at the tip of the cracks which furthers their growth. At the end as the surface roughness is severely increased, liquid material accumulates on the surface and reduces the impact damage of the oncoming drops, which explains the reduced mass loss rate in the placid period.

\subsection{CFD simulation of raindrop impact}

The impact velocity under investigation is $100 \mathrm{~m} / \mathrm{s}$. The impacts are normal to a rigid smooth surface. The drops are spherical and the impact velocity choice is based on the range of the blade tip velocities shown in Figure 2. The smoothness of the surface is justified since the goal is to predict the incubation time at the end of which the surface develops roughness. In order to better understand the impact modeling, it is useful to have a look at two important non-dimensional numbers relevant to the fluid dynamics of drop impact. In the case of a $100 \mathrm{~m} / \mathrm{s}$ impact, the Weber and Reynolds numbers of a $1 \mathrm{~mm}$ diameter water drop are approximately $134 \times 10^{3}$ and $112 \times 10^{3}$ respectively. It is evident that the fluid dynamics of such impacts is dominated by inertia and that surface tension and viscous effects are negligible in comparison. In what follows we describe the equations governing the dynamics of liquid drop impingement on solid target and the numerical methods to solve these equations.

\subsubsection{Governing equations}

The governing equations are the conservation of mass and momentum [48], which are:

$\nabla \cdot \vec{U}=0$

$\frac{\partial \vec{U}}{\partial t}+(\vec{U} \cdot \nabla) \vec{U}=-\frac{1}{\rho} \nabla p+\nu \nabla^{2} \vec{U}+\frac{1}{\rho} \overrightarrow{F_{b}}+\frac{1}{\rho} \overrightarrow{F_{S T}}$

where $\vec{U}$ is the velocity vector, $p$ is the pressure, $v$ is kinematic viscosity, $\rho$ is the density, $\vec{F}_{b}$ is the body force and $\vec{F}_{S T}$ is the surface tension force. The drop volume is tracked using the volume-of-fluid method [49], in which a scalar function $f$ is defined to represent the drop and is equal to one inside the drop and equal to zero outside. The following advection equation is solved to transport $f$ :

$\frac{\partial f}{\partial t}+(\vec{U} \cdot \nabla) f=0$

The unit vector normal to the drop surface $\hat{n}$ and the curvature of drop surface $\kappa$ are determined from $f$ via $\hat{n}=\nabla f /|\nabla f|$ and $\kappa=-\nabla \cdot(\nabla f /|\nabla f|)$.

\subsubsection{Numerical methods}

Two-step projection method [50] is used to solve Equations (8) and (9). In this method, a time discretization of Equation (9) is split into two steps shown below: 
$\frac{\vec{U}-\vec{U}^{n}}{\Delta t}=-\left(\vec{U}^{n} \cdot \vec{\nabla}\right) \vec{U}^{n}+v \nabla^{2} \vec{U}^{n}+\frac{1}{\rho^{n}} \vec{F}_{b}^{n}+\frac{1}{\rho^{n}} \vec{F}_{S T}^{n}$

$\frac{\vec{U}^{n+1}-\vec{U}}{\Delta t}=-\frac{1}{\rho^{n}} \nabla p^{n+1}$

where $\vec{U}$ is an interim velocity and the superscripts $n$ and $n+1$ denote two consecutive time levels. In the first step, the interim velocity is computed explicitly by considering the effects of the convective, viscous, and body and surface tension forces. The surface tension is applied by using the CSF method [51]. In the second step, Equation (8) is combined with Equation (12) at time level $n+1$ to obtain a pressure Poisson problem:

$\frac{1}{\Delta t}(-\nabla \cdot \vec{U})=-\nabla \cdot\left(\frac{1}{\rho^{n}} \vec{\nabla} p^{n+1}\right)$

The pressure field is obtained at each time step by solving Equation (13) iteratively. The new velocity field $\vec{U}^{n+1}$ is evaluated via Equation (12). The iterative solution of the pressure Poisson problem in Equation (13) is the most computationally intensive step of the entire simulation. To accelerate the simulations, we use an in-house GPUaccelerated pressure Poisson solver (SCGPU). It has been shown that SCGPU provides speed-ups as high as 15 times in the total simulation time of a three-dimensional case with 28 million grid points [52]. Equation (10) is solved geometrically using the Youngs' method [53] to track the drop volume. No-slip boundary condition is imposed on solid surfaces. The contact angle is imposed at the contact line, which is a function of the contact line velocity. The contact line velocity is approximated as the velocity parallel to the surface at half a cell above the surface, where the closest velocity information reside. The details of the contact angle model, which includes advancing, receding and equilibrium contact angles, are presented in [49, 54].

\subsection{Impact pressure calculations via multi-resolution analysis}

In order to take advantage of the symmetry of a normal impact, only a quarter of the drop was simulated to reduce the computational cost. The $100 \mathrm{~m} / \mathrm{s}$ normal impact of a family of raindrops, representative of the typical range of raindrop size in the rain scenarios (rain intensities) under consideration, are simulated. During the simulations the impact pressures are recorded at $1 \mathrm{~ns}$ intervals from the center of the first layer of cells on the impact surface in the form of radial distribution of pressure from the central axis of the drop. Initially, the simulations are performed with 40 computational cells across the drop's diameter (CPD). The grid resolution is then gradually increased to attain grid convergence in the impact pressure. The results are shown in Figure 12. The main features that are considered as convergence criteria are the maximum central and lateral pressure peaks. As the resolution is increased, several changes are observed in the pressure profile. The magnitudes of the central and lateral pressure peaks are increased. Also, the time evolution of the pressure profile is accelerated. The changes in the central and lateral peaks are noticeable at lower resolutions, for example, when moving from 40 to $80 \mathrm{CPD}$ but become smaller with each refinement step and are negligible at $360 \mathrm{CPD}$. Therefore, $360 \mathrm{CPD}$ is chosen as a suitable resolution for the simulations. However, 
in terms of the computational cost, simulating an entire impact at 360 CPD is very memory intensive and requires considerable processing power. Therefore, certain characteristics of the drop impact are considered to set up a feasible simulation.

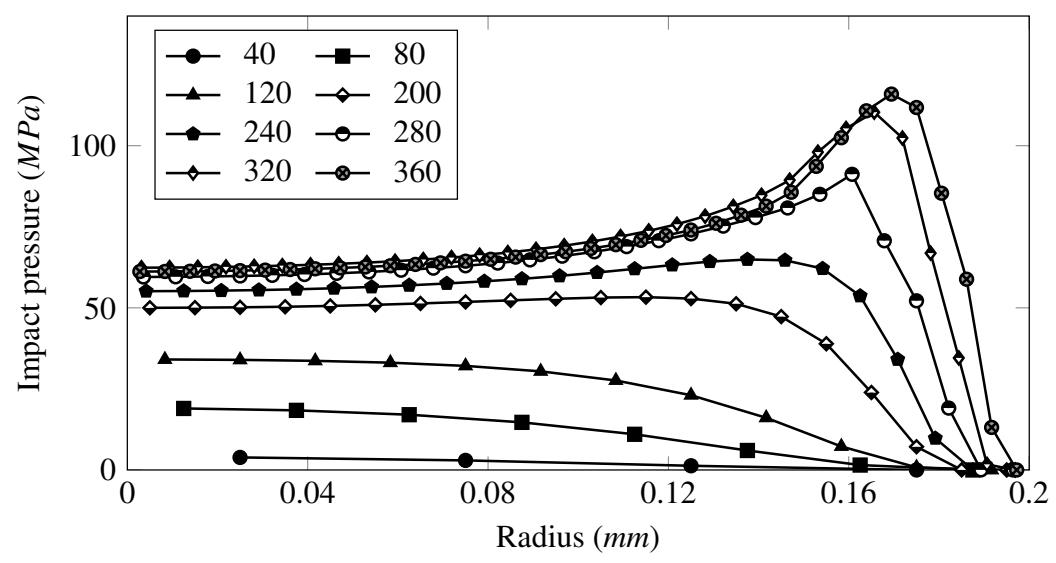

Figure 12: A snapshot of the impact pressure at various mesh resolutions from 40 to 360 cells per diameter. The diameter is $2 \mathrm{~mm}$.

In the grid convergence study, it is observed that as the grid resolution is increased, the changes in the pressure profile remain limited to the early stages of the impact. In other words, the central and lateral peaks are the only changing features of the profile. The sensitivity of the lateral pressure peak to the grid resolution has also been pointed out in [55]. In fact, the pressure profiles of different CPDs are matching from shortly after the occurrence of the lateral peak maximum. Therefore, it is not necessary to simulate the entire impact scenario at the highest resolution. The high cost $360 \mathrm{CPD}$ simulations can thus be limited to a time frame where central and lateral pressure peaks appear and the remainder of the impact can be simulated at a lower resolution. The 240 CPD resolution is chosen for this purpose.

Although the $360 \mathrm{CPD}$ simulation is now limited to the early stages of impact, the memory requirements are still overwhelming. Another property of the impact pressure is thus used to overcome this problem. The impact pressure develops due to the sudden change of the momentum in the drop. However, this change is local to the impact point, particularly in the early stages of impact when the central and lateral pressure peaks appear. Therefore, in the early stages of impact, the top portion of the drop does not even realize that an impact has occurred. In order to test this hypothesis, the impact is simulated with the top-half portion of the quarter drop removed, the result of which is shown in Figure 13 and compared against full quarter case. It is observed that the pressure profiles of the full quarter drop and the half quarter drop perfectly match, and discrepancies appear only after the occurrence of the lateral peak maximum. As a result, it is only necessary to simulate one-eighth of the drop at $360 \mathrm{CPD}$, which reduces the amount of the required memory by half. 


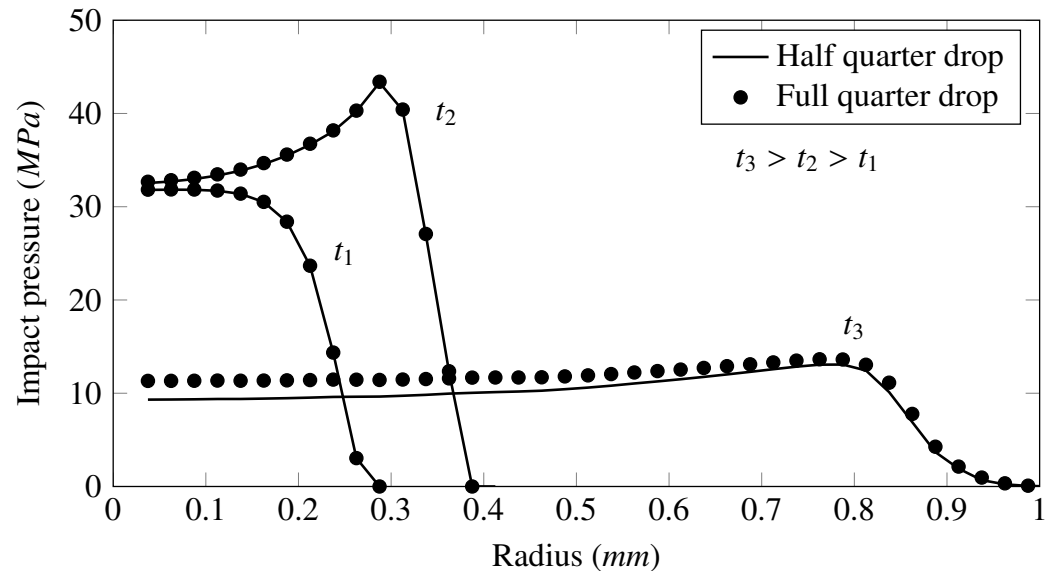

Figure 13: Comparison between the pressure profiles of a full quarter drop and a halved quarter drop at $80 \mathrm{CPD}$ resolution. The diameter is $2 \mathrm{~mm}$.

As mentioned earlier, in the final stages of impact, the pressure drops down to the stagnation pressure and finally goes to zero over time. In this time period, the pressure profile changes very slowly compared to the early stages of impact. Therefore, simulating this time period at 240 CPD can be a waste of computational resources. Also, it was mentioned that the pressure profiles at different resolutions begin to match shortly after the lateral peak maximum appears. Therefore, it is more convenient yet not inaccurate to simulate the final stages of impact at a lower resolution. The 40 CPD resolution is chosen for this purpose.

In summary, the full time dependent pressure profile for each drop size is obtained by combining together the results from the simulations at various resolutions, i.e. 360 CPD for a time period immediately after the impact, 240 CPD for the intermediate stages and 40 CPD for the final time period where the pressure profile dies out. Matching frames are chosen between different resolutions to act as transition from one resolution to another.

\subsection{Simulation results and validation}

There is no firm consensus among researchers on the estimate of the pressure induced due to impact of water drops on a surface. However, most agree that the impact pressure can be explained by the water hammer equation [56]:

$p=\rho_{l} c_{l} v_{0}$

where $p$ is pressure, $\rho_{l}$ is the liquid density, $c_{l}$ is the sonic velocity in the liquid and $v_{0}$ is the impact velocity. Considering a water density of $1000 \mathrm{~kg} / \mathrm{m}^{3}$ and sound velocity of $1400 \mathrm{~m} / \mathrm{s}$, the $100 \mathrm{~m} / \mathrm{s}$ impact of a water drop produces a $140 \mathrm{MPa}$ pressure. One of the earliest calculations of the impact pressure was performed by Engel in 1955 [57]. In this theoretical and experimental study, it is proposed that the average pressure during the spherical drop impact is given by:

$p=\frac{\beta}{2} \rho_{l} c_{l} v_{0}$ 
where $\beta / 2$ is a correction coefficient for the spherical shape of the drop. Note that the original water hammer equation given by Equation (14) is for the impact of a cylindrical body of water along its axis. The value of $\beta$ approaches unity as the impact velocity is increased [57]. By substituting the corresponding values for water, the average impact pressure cannot exceed $70 \mathrm{MPa}$. Generalized forms of the water hammer equation, accounting for the deformability of the solid target have also been proposed. An example is the following equation [45]:

$p=\frac{\rho_{l} c_{l} v_{0}}{1+\frac{\rho_{l} c_{l}}{\rho_{s} c_{s}}}$

where all parameters have the same definition as in Equation (14) and subscript $s$ denotes the properties of the deformable solid target. A quick look at these studies and similar ones in the literature reveals that, more often than not, a corrected version of the water hammer equation is used to estimate the impact pressure in liquid drop impact on solid target and less effort has been made to study the impact pressure behavior over time. The numerical study by Hwang and Hammit [58], for example, is one of the earliest works in which the transient impact pressure and its time evolution was investigated. For various liquid body geometries including spherical drops, in the case of a $300 \mathrm{~m} / \mathrm{s}$ impact, they reported that the maximum pressure on the central axis of the drop is approximately 0.7 water hammer pressure and that the maximum pressure overall is experienced near the contact line and is almost equal to the water hammer pressure. In the case of a $100 \mathrm{~m} / \mathrm{s}$ impact, these pressures are 98 and $140 \mathrm{MPa}$ respectively. Figure 14 shows snapshots of the impact pressures extracted from 1 to $5 \mathrm{~mm}$ drop simulations. The solid and dashed lines represent the pressure profiles at maximum central and lateral pressure peaks respectively which are equal to 65 and $120 \mathrm{MPa}$ and match the range reported in the literature. Furthermore, it is seen in Figure 14 that drops with various diameters lead to the same impact pressure magnitudes, but obviously different spread areas, which is again in agreement with the observations reported in [55].

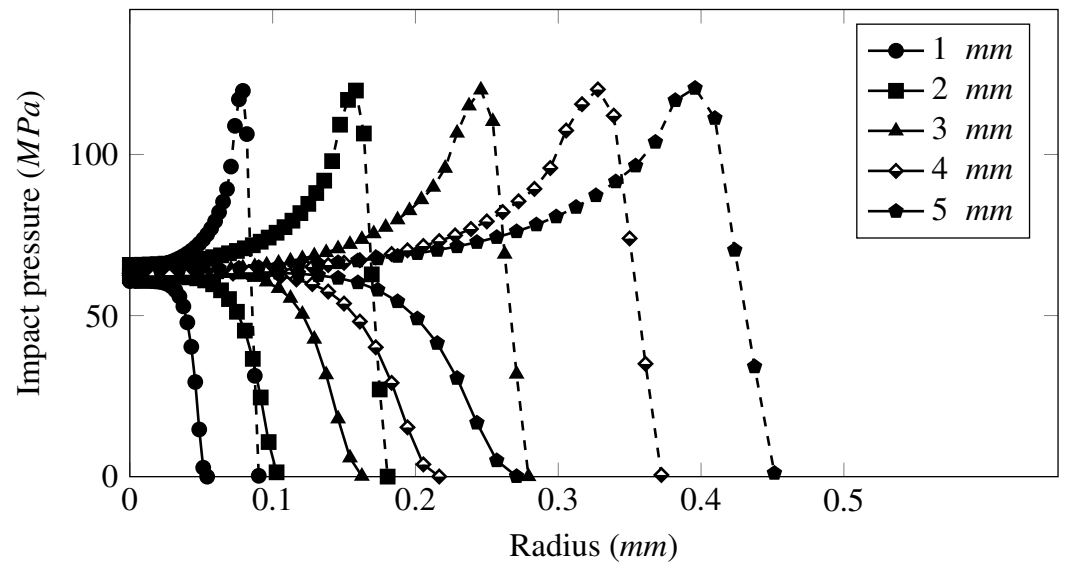

Figure 14: Maximum central and lateral pressure peaks obtained from the impact simulations of 1 to $5 \mathrm{~mm}$ drops. The solid and dashed lines represent the maximum central and lateral pressure peaks respectively. The impact velocity is $100 \mathrm{~m} / \mathrm{s}$. 


\section{Impact pressure interpolation}

As mentioned earlier, the drop impact simulations are computationally expensive and performing a large number of them to simulate a rain event is simply not feasible. In addition, it is also wasteful to simulate the impact for all drop sizes in a sample, since a new sample would require an entirely new set of simulations. Therefore, impact simulations are instead performed for a representative set of raindrop sizes. The pressure profiles and their evolution over time extracted from these simulations are then used to create a library of impact pressures which is subsequently fed into an interpolation scheme to calculate the impact pressure profile for raindrops of arbitrary size.

In order to explain the interpolation scheme, let us assume that the pressure profile of a $2.5 \mathrm{~mm}$ drop at $300 \mathrm{~ns}$ after impact is required. Assuming that the library contains the pressure profiles for drops of size 1 to $5 \mathrm{~mm}$ at $1 \mathrm{~ns}$ intervals, the following steps are performed in the interpolation scheme:

1. Radial normalization of the pressure profiles in the library: It was seen in the previous section that as the drop size is increased, the evolution of the impact pressure slows down, i.e. larger drops reach the lateral pressure peak maximum later than the smaller ones. In addition, at a given instant in time, say $300 \mathrm{~ns}$, the pressure profile of smaller drops is spread over a smaller area compared to the larger drops. Therefore, we normalize the pressure profiles in the library by their spread radius at $300 \mathrm{~ns}$, resulting in pressure profiles that are spread over $[0,1]$ interval no matter what the size of the drop is (see Figure 15).
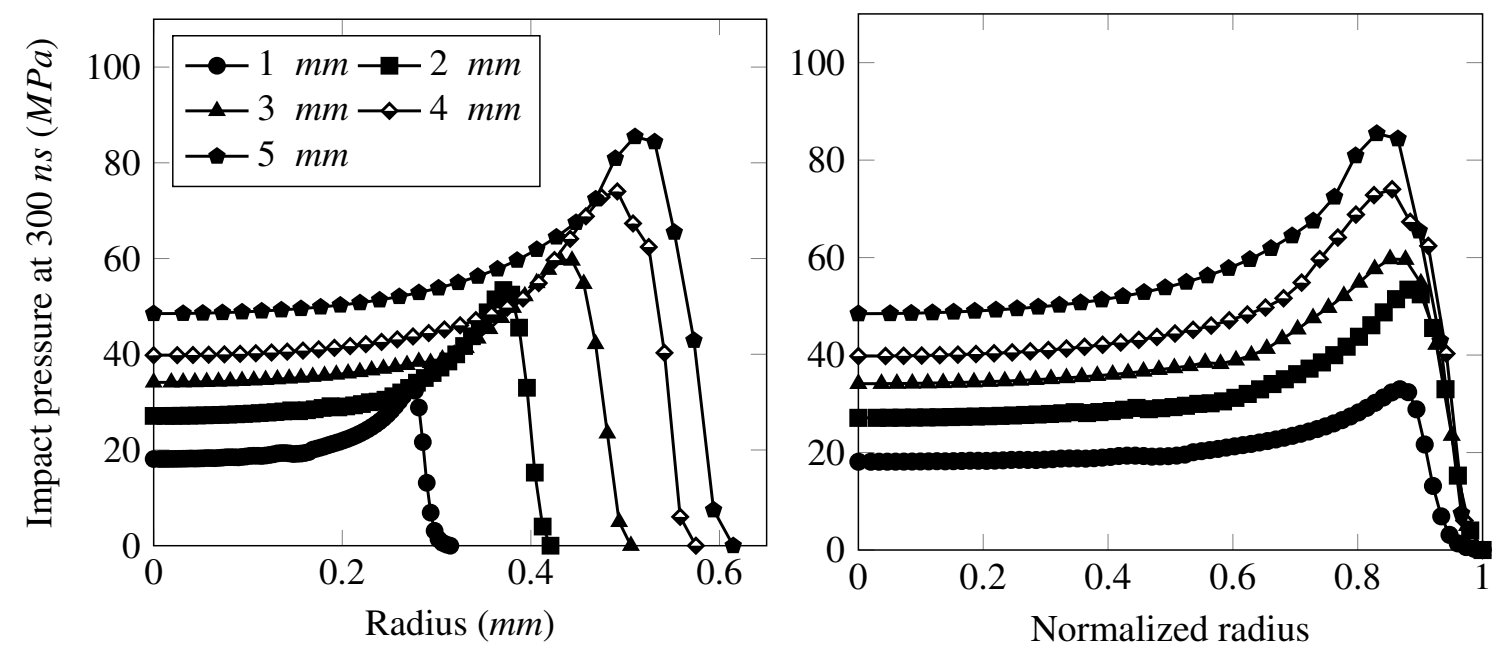

Figure 15: Impact pressure versus spread radius at $300 \mathrm{~ns}$ after impact for raindrop diameters varying between 1 to $5 \mathrm{~mm}$, impacting at $100 \mathrm{~m} / \mathrm{s}$ (Left). The same impact pressures plotted against a normalized radius (Right). The normalized radius for each drop size is obtained by dividing the radius axis by the spread radius of each drop.

2. Radial interpolation within individual pressure profiles in the library: In order for the interpolation to succeed, all library pressure profiles must have equal number of data points along the normalized radius. This is achieved through a radial linear interpolation within individual pressure profiles in the library. This procedure is shown in Figure 16 for a $3 \mathrm{~mm}$ drop as an example. As shown in the figure, using the above interpolation, the 
pressure profile of the $3 \mathrm{~mm}$ drop is regenerated to match the number of data points and their radial positions corresponding to the smallest drop size, i.e., $1 \mathrm{~mm}$ in this case.

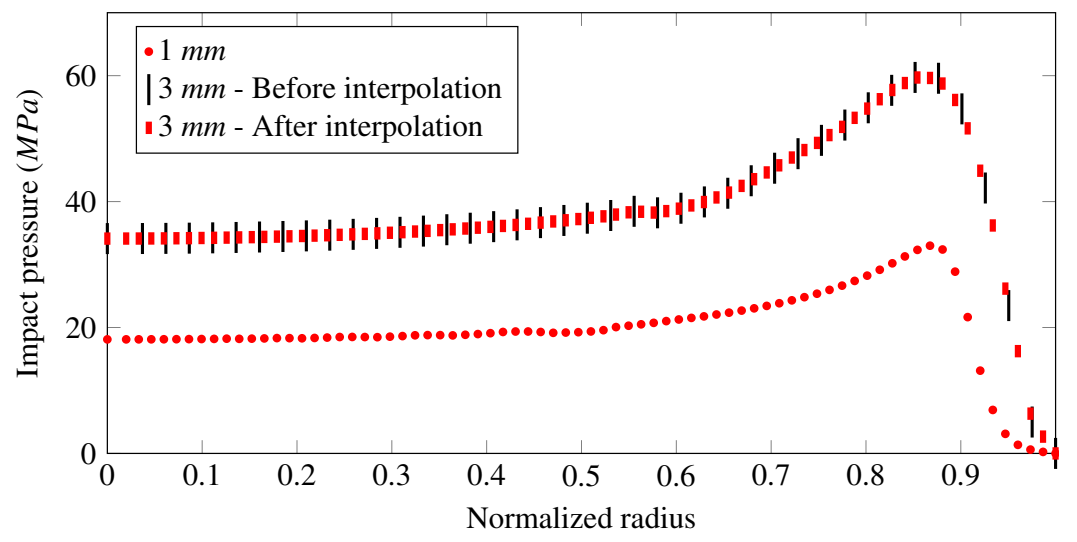

Figure 16: Linear interpolation within individual pressure profiles in the impact pressure library.

3. Pressure interpolation based on drop size: The next step is to use the normalized pressure profiles stored in the library to obtain the normalized pressure profile for a desired drop size at a given instant in time. This is done through a linear interpolation and for all the points along the normalized radius (see Figure 17).

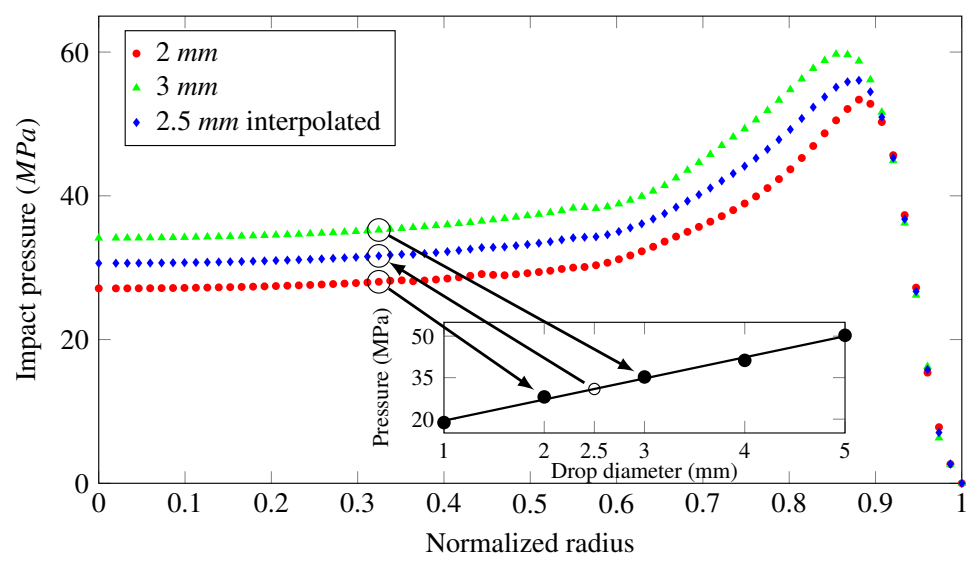

Figure 17: Linear pressure interpolation based on drop size.

4. Radial expansion of the interpolated normalized profile: The last step is to transform the interpolated profile in the normalized radius space to the actual pressure profile. This is done by multiplying the coordinates of the points along the normalized radius by the spread radius for the drop size under consideration. This spread radius is interpolated from the spread radii of the drops in the library.

Figure 18 depicts the comparison between pressure profiles obtained via high fidelity CFD simulations and the procedure described above for a $2.5 \mathrm{~mm}$ drop at $t=300 \mathrm{~ns}$ as an example. It is seen that the proposed procedure is able to reproduce the pressure profile with great accuracy and the results of the interpolation scheme practically match those 
obtained via high fidelity CFD simulations. It should be noted that, even with the help of GPU acceleration, the CFD simulations can sometimes take up to a few days (2-3 days) as they involve a large amount of unnecessary overhead computations not relevant to the evolution of the pressure profile. The proposed interpolation scheme, on the other hand, drastically reduces the computation time of pressure profile calculations to less than a few minutes on the same hardware.

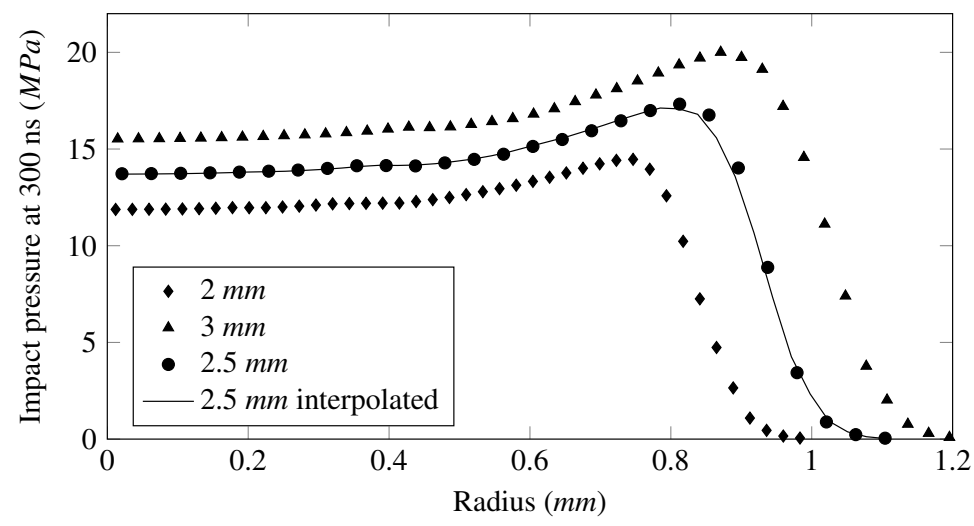

Figure 18: Comparison between the interpolated and the simulated pressure profiles of a $2.5 \mathrm{~mm}$ raindrop. The pressure profiles of the 2 and $3 \mathrm{~mm}$ drops used in the interpolation process are also shown for comparison.

\section{Concluding remarks}

This paper is the first part in a two-part paper that develops a computational framework for rain erosion prediction in wind turbine blades by integrating several ingredients based in probability theory and stochastic analysis, multiresolution computational fluid dynamics, transient finite element modeling, and fatigue analysis. In this part, we were concerned with two fundamental ingredients of modeling rain-induced erosion, that is modeling the rain texture and simulation of raindrop impingement on solid objects. We aimed at demonstrating how a general framework that attempts to model the complex process of rain erosion involving the random rain and multiple raindrop impacts can benefit from rigorous stochastic analysis, and efficient yet accurate multi-resolution and interpolation-based surrogate modeling approaches to fluid dynamics simulations. A stochastic model of rain texture was developed that is capable of relating the integral properties of rain such as rain intensity and average volume of water per unit volume of air to its micro-structural properties such as raindrop size and spatial distribution. In particular, the proposed model establishes a relationship between the rain intensity and the number of raindrops per unit volume of air and allows for reproducing three-dimensional fields of raindrops through a compound stochastic point process. The spatial and temporal variations of the impact pressure in the droplet-surface collision was calculated using an in-house GPUaccelerated CFD model of free-surface flows. In order to reduce the computational cost, a multi-resolution approach was proposed. In the proposed approach computationally intensive high resolution simulations were restricted to the time period of utmost importance that is the time frame before central and lateral pressure peaks develop. The 
remainder of the impact was then simulated with intermediate and low resolutions for the intermediate and final stages of pressure profile evolution. In addition, an interpolation scheme was developed to calculate the impact pressure profile for any raindrop size accurately and efficiently using the results of a few representative droplet impact simulations.

\section{Acknowledgements}

This work was supported by a grant from the University of Massachusetts President's Office under the Science and Technology Initiative Program. This support is gratefully acknowledged. M. Raessi also gratefully acknowledges support from the National Science Foundation under the CBET Grant No. 1336232.

\section{References}

[1] U.S. Energy Information Administration, International Energy Outlook 2011, Tech. rep., U.S. Energy Information Administration (2011) URL http://www.eia.doe.gov/oiaf/ieo/index.html

[2] Global Wind Energy Council, Global Wind Report, Tech. rep. (2012).

[3] 4C Offshore, Vestas v164 wind turbine product specifications (2014). URL http://goo.gl/3vgT1U

[4] 4C Offshore, Samsung s7.0-171 wind turbine product specifications (2014). URL http://goo.gl/HRQfZ2

[5] K. Wood, Blade repair: Closing the maintenance gap, Composites Technology (2011).

[6] G. Marsh, Meeting the challenge of wind turbine blade repair, Reinforced Plastics 55 (4) (2011) 32-36.

[7] N. Dalili, A. Edrisy, R. Carriveau, A review of surface engineering issues critical to wind turbine performance, Renewable and Sustainable Energy Reviews 13 (2) (2009) 428-438.

[8] H. Macdonald, D. Infield, D. H. Nash, M. M. Stack, Mapping hail meteorological observations for prediction of erosion in wind turbines, Wind Energy (2015) (In press).

[9] Intergovernmental Panel on Climate Change, Special Report on Renewable Energy Sources and Climate Change Mitigation, Tech. rep., Cambridge Unviersity Press (2012).

[10] A. Sareen, C. A. Sapre, M. S. Selig, Effects of leading edge erosion on wind turbine blade performance, Wind Energy 17 (10) (2014) $1531-1542$.

[11] Y. Uematsu, M. Yamada, Effects of aspect ratio and surface roughness on the time-averaged aerodynamic forces on cantilevered circular cylinders at high reynolds numbers, Journal of wind engineering and industrial aerodynamics 54 (1995) 301-312.

[12] G. Matteoni, C. Georgakis, Effects of bridge cable surface roughness and cross-sectional distortion on aerodynamic force coefficients, Journal of Wind Engineering and Industrial Aerodynamics 104 (2012) 176-187.

[13] G. Matteoni, C. T. Georgakis, Effects of surface roughness and cross-sectional distortion on the wind-induced response of bridge cables in dry conditions, Journal of Wind Engineering and Industrial Aerodynamics 136 (2015) 89-100.

[14] W. Chakroun, I. Al-Mesri, S. Al-Fahad, Effect of surface roughness on the aerodynamic characteristics of a symmetrical airfoil, Wind Engineering 28 (5) (2004) 547-564.

[15] F. Schuerich, R. E. Brown, Effect of dynamic stall on the aerodynamics of vertical-axis wind turbines, AIAA journal 49 (11) (2011) 25112521.

[16] K. Almohammadi, D. Ingham, L. Ma, M. Pourkashanian, Modeling dynamic stall of a straight blade vertical axis wind turbine, Journal of Fluids and Structures 57 (2015) 144-158 
[17] P. Pourazarm, L. Caracoglia, M. Lackner, Y. Modarres-Sadeghi, Stochastic analysis of flow-induced dynamic instabilities of wind turbine blades, Journal of Wind Engineering and Industrial Aerodynamics 137 (2015) 37-45.

[18] L. Rempel, Rotor blade leading edge erosion-real life experiences, Wind Systems Magazine (2012).

[19] S. Stephenson, Wind blade repair: Planning, safety, flexibility, Composites World (2011).

URL http://goo.gl/JAuwfm

[20] J. D. Sørensen, Framework for risk-based planning of operation and maintenance for offshore wind turbines, Wind energy 12 (5) (2009) 493-506.

[21] X. Zhang, L. Sun, H. Sun, Q. Guo, X. Bai, Floating offshore wind turbine reliability analysis based on system grading and dynamic fta, Journal of Wind Engineering and Industrial Aerodynamics 154 (2016) 21-33.

[22] M. H. Keegan, D. H. Nash, M. M. Stack, On erosion issues associated with the leading edge of wind turbine blades, Journal of Physics D: Applied Physics 46 (38) (2013) 383001.

[23] H. J. Sutherland, A summary of the fatigue properties of wind turbine materials, Wind energy 3 (1) (2000) 1-34.

[24] P. Giguère, M. S. Selig, Aerodynamic effects of leading-edge tape on aerofoils at low reynolds numbers, Wind Energy 2 (3) (1999) 125-136.

[25] O. Gohardani, Impact of erosion testing aspects on current and future flight conditions, Progress in Aerospace Sciences 47 (4) (2011) $280-303$.

[26] H. W. Bargmann, On the time-dependence of the erosion rate: A probabilistic approach to erosion, Theoretical and Applied Fracture Mechanics 6 (3) (1986) 207-215.

[27] M. H. Keegan, D. H. Nash, M. M. Stack, Modelling rain drop impact of offshore wind turbine blades, in: ASME Turbo Expo 2012: Turbine Technical Conference and Exposition, American Society of Mechanical Engineers, 2012, pp. 887-898.

[28] E. Yarrapareddy, R. Kovacevic, Numerical simulation and characterization of slurry erosion of laser cladded surfaces by using failure analysis approach, Journal of Failure Analysis and Prevention 7 (6) (2007) 464-474.

[29] G. Salomon, Application of systems thinking to tribology, ASLE transactions 17 (4) (1974) 295-299.

[30] H. M. Slot, E. R. M. Gelinck, C. Rentrop, E. van der Heide, Leading edge erosion of coated wind turbine blades: Review of coating life models, Renewable Energy 80 (2015) 837-848.

[31] Z. Wang, Y. Zhao, F. Li, J. Jiang, Extreme dynamic responses of MW-level wind turbine tower in the strong typhoon considering wind-rain loads, Mathematical Problems in Engineering (2013) 1-13.

[32] B. Amirzadeh, A. Louhghalam, M. Raessi, M. Tootkaboni, A computational framework for rain erosion prediction in wind turbine blades, part II: drop impact-induced stress analysis and blade coating fatigue life, Journal of Wind Engineering \& Industrial Aerodynamics (Under review).

[33] X. Fu, H.-N. Li, Y. Yang, Calculation of rain load based on single raindrop impinging experiment and applications, Journal of Wind Engineering and Industrial Aerodynamics 147 (2015) 85-94.

[34] National Oceanic and Atmospheric Administration, Climate data online: Dataset discovery (2013). URL http://www.ncdc.noaa.gov/cdo-web/datasets

[35] A. C. Best, The size distribution of raindrops, Quarterly Journal of the Royal Meteorological Society 76 (327) (1950) 16-36.

[36] J. S. Marshall, W. M. K. Palmer, The distribution of raindrops with size, Journal of Meteorology 5 (4) (1948) $165-166$.

[37] M. C. Hodson, Raindrop size distribution, Journal of Climate and Applied Meteorology 25 (7) (1986) 1070-1074.

[38] J. O. Laws, D. A. Parsons, The relation of raindrop-size to intensity, Transactions, American Geophysical Union 24 (1943) $452-460$.

[39] C. W. Ulbrich, Natural variations in the analytical form of the raindrop size distribution, Journal of Climate and Applied Meteorology 22 (10) (1983) 1764-1775.

[40] N. G. Van Kampen, Stochastic processes in physics and chemistry, Elsevier, 1992.

[41] A. B. Kostinski, M. L. Larsen, A. R. Jameson, The texture of rain: Exploring stochastic micro-structure at small scales, Journal of Hydrology 328 (1) (2006) 38-45.

[42] A. B. Kostinski, A. R. Jameson, On the spatial distribution of cloud particles, Journal of the Atmospheric Sciences 57 (7) (2000) $901-915$.

[43] L. Devroye, Nonuniform random variate generation, Handbooks in Operations Research and Management Science 13 (2006) $83-121$. 
[44] G. S. Springer, C. I. Yang, Model for the rain erosion of fiber reinforced composites, AIAA Journal 13 (7) (1975) $877-883$.

[45] W. F. Adler, The mechanics of liquid impact, Academic Press, Treatise on Materials Science and Technology, 16 (1979) $127-183$.

[46] C. M. Preece, N. H. Macmillan, Erosion, Annual Review of Materials Science 7 (1) (1977) 95-121.

[47] G. P. Thomas, J. H. Brunton, Drop impingement erosion of metals, Proceedings of the Royal Society of London. A. Mathematical and Physical Sciences 314 (1519) (1970) 549-565.

[48] M. Bussmann, S. Chandra, J. Mostaghimi, Modeling the splash of a droplet impacting a solid surface, Physics of Fluids 12 (12) (2000) 3121-3132

[49] M. Bussmann, J. Mostaghimi, S. Chandra, On a three-dimensional volume tracking model of droplet impact, Phys. Fluids 11 (1999) 14061417.

[50] A. J. Chorin, Numerical Solution of the Navier-Stokes Equations, Mathematics of Computation 22 (104) (1968) $745-762$.

[51] J. U. Brackbill, D. B. Kothe, C. Zemach, A continuum method for modeling surface tension, J. Comput. Phys. 100 (1992) 335-354.

[52] S. Codyer, M. Raessi, M. Khanna, Using graphics processing units to accelerate numerical simulations of interfacial incompressible flows, in: ASME 2012 Fluids Engineering Summer Meeting, Rio Grande, Puerto Rico, 2012.

[53] D. L. Youngs, An interface tracking method for a 3D Eulerian hydrodynamics code, Tech. Rep. 44/92/35, AWRE (1984).

[54] M. Pasandideh-Fard, S. Chandra, J. Mostaghimi, A three-dimensional model of droplet impact and solidification, Int. J. Heat Mass Transfer 45 (2002) 2229-2242.

[55] J. Xiong, S. Koshizuka, M. Sakai, H. Ohshima, Investigation on droplet impingement erosion during steam generator tube failure accident, Nuclear Engineering and Design 249 (2012) 132-139.

[56] M. B. Lesser, Thirty years of liquid impact research: a tutorial review, Wear 186 (1995) 28-34.

[57] O. G. Engel, Waterdrop collisions with solid surfaces, Journal of Research of the National Bureau of Standards 54 (5) (1955) 281-298.

[58] J. B. G. Hwang, F. G. Hammitt, High-speed impact between curved liquid surface and rigid flat surface, Journal of Fluids Engineering 99 (2) (1977) 396-404. 